\title{
Adropin is associated with hyperhomocysteine and coronary atherosclerosis
}

\author{
LIANG-PING ZHAO ${ }^{1}$, TAO YOU ${ }^{1}$, SIEW-PANG CHAN ${ }^{2-4}$, JIAN-CHANG CHEN ${ }^{1}$ and WEI-TING XU ${ }^{1}$ \\ ${ }^{1}$ Department of Cardiology, The Second Affiliated Hospital of Soochow University, Suzhou, Jiangsu 215004, P.R. China; \\ ${ }^{2}$ Department of Medicine, Yong Loo Lin School of Medicine, National University of Singapore, Singapore 117597; \\ ${ }^{3}$ Cardiovascular Research Institute, National University Heart Centre Singapore, National University Health System, \\ Singapore 119228, Republic of Singapore; ${ }^{4}$ Department of Mathematics and Statistics, \\ School of Engineering and Mathematical Sciences, Faculty of Engineering, Technology and Science, \\ La Trobe University, Melbourne, Victoria 3086, Australia
}

Received October 26, 2014; Accepted November 23, 2015

DOI: $10.3892 /$ etm.2015.2954

\begin{abstract}
Homocysteine has been recognized as a risk factor for atherosclerosis and cardiovascular diseases. Adropin is a newly-identified energy homeostasis protein with a potential protective effect against coronary artery disease (CAD). This study attempted to measure the correlation between serum homocysteine and adropin levels in patients with CAD, and to ascertain how the two hormones could affect the severity of coronary atherosclerosis. A cohort of CAD patients who had undergone coronary angiography was prospectively recruited. The serum homocysteine and adropin levels of the patients were measured and the severity of coronary atherosclerosis was quantified with the SYNTAX score. The data were analyzed with a generalized structural equation model. In total, 170 consecutive patients were recruited with a mean serum homocysteine level of $15.9 \pm 8.3 \mu \mathrm{mol} / 1$, and $76(44.7 \%)$ patients were identified as hyperhomocysteinemic with a serum homocysteine level $>15 \mu \mathrm{mol} / 1$. Serum homocysteine level was found to be significantly negatively correlated with serum adropin level ( $\mathrm{r}=-0.169, \mathrm{P}=0.028)$. Patients with hyperhomocysteinemia had lower serum adropin levels and higher SYNTAX scores than patients without hyperhomocysteinemia. Further analysis with a generalized structural equation model showed that adropin was significantly associated with hyperhomocysteinemia (adjusted odds ratio: 0.95, 95\% confidence interval: 0.93 to $0.98 ; \mathrm{P}=0.002$ ), which in turn was significantly associated with the SYNTAX score (coefficient: 4.71, $95 \%$ confidence interval: 1.39 to $8.03 ; \mathrm{P}=0.005)$. In conclusion,
\end{abstract}

Correspondence to: Dr Wei-Ting Xu, Department of Cardiology, The Second Affiliated Hospital of Soochow University, 1055 Sanxiang Road, Suzhou, Jiangsu 215004, P.R. China

E-mail: xuwt1968@sina.com

Key words: adropin, homocysteine, atherosclerosis, SYNTAX score, correlation the serum homocysteine level was inversely correlated with the serum adropin level in patients with CAD. A low serum adropin level was associated with hyperhomocysteinemia and more severe coronary atherosclerosis, as reflected by a higher SYNTAX score.

\section{Introduction}

Homocysteine, a sulfur-containing amino acid produced in the metabolism of the essential amino acid methionine, has been recognized as a potential risk factor for atherosclerosis and cardiovascular diseases $(1,2)$. Epidemiological studies have shown that hyperhomocysteinemia, characterized by a serum homocysteine level $>15 \mu \mathrm{mol} / 1$, is not only associated with the onset of coronary artery disease (CAD) but also can predict the mortality of patients with CAD $(3,4)$. Recent studies have reported that the blood homocysteine level is significantly associated with calcified plaque and the severity of CAD, assessed by computed tomography angiography and the Gensini scoring system (5-7). While there is published evidence supporting the pathophysiologic mechanisms linking homocysteine to atherosclerosis, including an increase of oxidative stress and promotion of the oxidation of low-density lipoprotein (8), stimulation of vascular smooth muscle cell proliferation $(9,10)$ and inducement of vascular inflammation and endothelial dysfunction (11), certain studies did not support the predictive effect of homocysteine on atherothrombotic cardiovascular diseases (12-14).

Adropin is a newly-identified secretary protein that participates in the regulation of energy homeostasis and insulin response. It is encoded by the energy homeostasis associated gene (Enho) and expressed in the liver, brain and endothelial cells $(15,16)$. The governing effects of adropin on glucose and lipid homeostasis could play an important role in the prevention of insulin resistance, impaired glucose tolerance, and dyslipidemia associated with diabetes and obesity $(15,17)$. In addition to the impact on body energy metabolism, there is also growing published evidence that adropin is a marker of cardiovascular diseases. An in vitro laboratory experi- 
ment showed that adropin-treated endothelial cells exhibited greater proliferation, migration, capillary-like tube formation and upregulation of the expression of endothelial nitric oxide synthase (eNOS) (16). Furthermore, previous studies have also reported that a lower serum adropin level is associated with acute myocardial infarction and the angiographic severity of coronary atherosclerosis $(18,19)$.

Since homocysteine and adropin have opposite effects on endothelial function and atherosclerotic disease, there could be a possible linkage between these two molecules. Thus far, there is a shortage of published evidence evaluating the relationship between homocysteine and adropin. In this exploratory study, the correlation between serum homocysteine and adropin levels in patients with CAD and the effects of the two hormones on the severity of coronary atherosclerosis (assessed with a SYNTAX scoring system) were investigated.

\section{Materials and methods}

Study design and subjects. This was a prospective observational study. Consecutive patients hospitalized at the Second Affiliated Hospital of Soochow University (Suzhou, China) for the diagnosis and management of CAD were recruited. To confirm the diagnosis of CAD, only patients who had undergone coronary angiography and were found to have $\geq 50 \%$ stenosis in at least one coronary artery were deemed eligible. Patients with end-stage renal failure, severe hepatic insufficiency, current microbial infections or malignant tumor were excluded. Details about the study protocol were explained to the eligible patients and informed consent was obtained. The study protocol was approved by the Medical Ethics Committee of the Second Affiliated Hospital of Soochow University.

Laboratory investigations. Samples of venous blood were collected following overnight fasting, and stored at $-80^{\circ} \mathrm{C}$ prior to analyses. Serum homocysteine level was measured by an enzymatic conversion method (Formosa Biomedical Technology Corporation, Taiwan); serum adropin level with a commercial enzyme-linked immunosorbent assay (ELISA) kit (JRDUN Biotechnology Co., Ltd., Shanghai, China); high sensitivity C-reactive protein (hsCRP) level with a high-sensitivity ELISA kit (Orion Diagnostica Oy, Espoo, Finland), and fasting plasma glucose with the hexokinase method (AU5400 High-Volume Chemistry Immuno Analyzer; Olympus Corporation, Tokyo, Japan). Serum creatinine level and serum lipid profiles including triglyceride, total cholesterol, low-density lipoprotein cholesterol and high-density lipoprotein cholesterol were assessed using standard methods. In brief, serum creatinine level was measured using the muscle amino acid oxidase method. Serum total cholesterol and triglyceride levels were measured by automated enzymatic procedures. The low-density lipoprotein cholesterol and highdensity lipoprotein cholesterol levels were determined after separating the lipoprotein fractions from fresh fasting sera by sequential ultracentrifugation, using the AU5400 analyzer.

Demographic, clinical and angiographic data collection. The demographic and clinical characteristics of the recruited patients were collected from hospital case records. These included age, gender, cigarette smoking status, hypertension, diabetes mellitus, and principal diagnosis at the index admission. Echocardiograms were carried out to determine the left ventricular ejection fraction prior to coronary angiography. Patients' height $(\mathrm{m})$ and weight $(\mathrm{kg})$ in light clothing were measured, and the body mass index $\left(\mathrm{kg} / \mathrm{m}^{2}\right)$ was calculated.

Coronary angiography was performed using standard techniques through radial or femoral approaches. Angiographic analysis was carried out by two experienced interventional cardiologists who were blinded to the study. The number of diseased coronary arteries (luminal diameter narrowing $\geq 50 \%$ ) was recorded, and patients with stenosis of the left main coronary artery $\geq 50 \%$ were considered to have two-vessel disease. SYNTAX scores of the study patients were calculated according to the guidelines of the SYNTAX Steering Committee and Boston Scientific Corporation (http:// www.syntaxscore.com/).

Statisticalanalyses.Exploratory analysesinvolving quantitative and qualitative data were performed with independent-sample t-tests (or the nonparametric Wilcoxon-Mann-Whitney tests if the data were non-normally distributed) and Chi-square tests, respectively. The association between homocysteine level, adropin level and SYNTAX score were assessed by pairwise Pearson correlation. In the following confirmatory analyses, a generalized structural equation model (gSEM) (20) was built with multiple risk factors and confounders in order to ascertain their effects on hyperhomocysteinemia (HyperHCY) and SYNTAX scores (SYNTAX). Patients were classified as suffering from hyperhomocysteinemia if their serum homocysteine level was $>15 \mu \mathrm{mol} / 1$ (21,22). The specific variables considered in the gSEM included demographic, clinical and angiographic characteristics. The details are depicted in Table I.

The proposed gSEM model, unlike the conventional regression, can accommodate multiple outcomes and handle more complex inter-relationships among the variables. It is a system of related models combined and estimated within a single framework, which is able to estimate the direct, indirect and total effects of the variables on the outcomes. As there were two outcomes of interest, the proposed gSEM model was constructed with two equations. SYNTAX was dealt with by a linear regression, and HyperHCY was analyzed with a binomial distribution and logit link in view of its binary nature. The data were exported to Stata/MP version 13 (Stata Corporation, College Station, TX, USA) for analysis, and all statistical tests were conducted with a $5 \%$ level of significance.

\section{Results}

Demographic, clinical and angiographic characteristics. Between July 2012 and May 2013, 170 consecutive patients with a mean serum homocysteine level of $15.9 \pm 8.3 \mu \mathrm{mol} / 1$, were successfully recruited into the study. The patients' demographic, clinical and angiographic characteristics are presented in Table I. Among the recruited patients, 76 (44.7\%) had suffered from hyperhomocysteinemia (serum homocysteine level $>15 \mu \mathrm{mol} / \mathrm{l})$. When compared with patients without the condition, they were found to have a lower serum adropin level, but higher body mass index, SYNTAX score and creatinine level. In addition, patients with hyperhomocysteinemia were 
Table I. Demographic, clinical and angiographic characteristics.

\begin{tabular}{|c|c|c|c|}
\hline Characteristics & $\begin{array}{l}\text { Nonhyperhomocysteinemia } \\
\qquad(\mathrm{n}=94)\end{array}$ & $\begin{array}{l}\text { Hyperhomocysteinemia } \\
\qquad(\mathrm{n}=76)\end{array}$ & P-value \\
\hline Age (year) & $64.6 \pm 11.5$ & $64.8 \pm 11.6$ & 0.923 \\
\hline Male, $\mathrm{n}(\%)$ & $62(66.0)$ & $54(71.1)$ & 0.511 \\
\hline Smoking, n (\%) & $44(46.8)$ & $39(51.3)$ & 0.644 \\
\hline Hypertension, $\mathrm{n}(\%)$ & $60(63.8)$ & $59(77.6)$ & 0.116 \\
\hline Diabetes, n (\%) & $17(18.1)$ & $18(23.7)$ & 0.491 \\
\hline Body mass index $\left(\mathrm{kg} / \mathrm{m}^{2}\right)$ & $23.7 \pm 3.1$ & $25.0 \pm 4.3$ & 0.033 \\
\hline Left ventricular ejection fraction $(\%)$ & $61.8 \pm 7.5$ & $55.3 \pm 11.4$ & 0.054 \\
\hline Adropin (pg/ml) & $75.9 \pm 55.7$ & $52.7 \pm 13.0$ & $<0.001$ \\
\hline Fasting plasma glucose $(\mathrm{mmol} / \mathrm{l})$ & $5.8 \pm 1.5$ & $6.7 \pm 1.9$ & 0.104 \\
\hline Cholesterol (mmol/l) & $5.0 \pm 2.4$ & $4.7 \pm 2.9$ & 0.603 \\
\hline Triglyceride $(\mathrm{mmol} / \mathrm{l})$ & $1.8 \pm 1.7$ & $2.1 \pm 3.0$ & 0.559 \\
\hline Low-density lipoprotein cholesterol (mmol/l) & $2.6 \pm 0.9$ & $2.7 \pm 1.1$ & 0.619 \\
\hline High-density lipoprotein cholesterol (mmol/l) & $1.1 \pm 0.3$ & $1.0 \pm 0.3$ & 0.436 \\
\hline Creatinine $(\mu \mathrm{mol} / \mathrm{l})$ & $68.6 \pm 22.1$ & $79.3 \pm 30.8$ & 0.029 \\
\hline hsCRP (mg/l) & $12.4 \pm 23.2$ & $18.9 \pm 37.4$ & 0.282 \\
\hline Clinical presentations, $\mathrm{n}(\%)$ & & & 0.614 \\
\hline STEMI & $24(25.5)$ & $23(30.3)$ & \\
\hline NSTEMI & $17(18.1)$ & $15(19.7)$ & \\
\hline Unstable angina & $31(33.0)$ & $18(23.7)$ & \\
\hline Stable angina/silent ischemia & $22(23.4)$ & $20(26.3)$ & \\
\hline Diseased vessel number, $\mathrm{n}(\%)$ & & & 0.038 \\
\hline 1-vessel & $43(45.7)$ & $22(28.9)$ & \\
\hline 2-vessel & $25(26.6)$ & $21(27.6)$ & \\
\hline 3-vessel & $26(27.7)$ & $33(43.4)$ & \\
\hline SYNTAX score & $19.1 \pm 10.3$ & $24.5 \pm 11.6$ & 0.002 \\
\hline
\end{tabular}

Data are mean \pm standard deviation or number $(\%)$. hsCRP, high sensitivity C-reactive protein; STEMI, ST-segment elevation myocardial infarction; NSTEMI, non-ST-segment elevation myocardial infarction. $\mathrm{P}$-values in bold are considered significant $(\mathrm{P}<0.05)$.

more likely to have 3-vessel disease, and less likely to have single vessel disease. No significant differences in age, gender, smoking status, hypertension, diabetes, left ventricular ejection fraction, fasting plasma glucose, cholesterol, triglyceride, low density lipoprotein cholesterol, high density lipoprotein cholesterol, hsCRP and principal clinical presentations were observed between the two groups.

Pairwise correlation. The recruited patients reported a mean SYNTAX score of 21.5 \pm 11.2 (range, 4-57). The serum homocysteine level was significantly negatively correlated with the serum adropin level (Pearson correlation $\mathrm{r}=-0.169, \mathrm{P}=0.028$ ). Although the serum homocysteine level was not significantly correlated with SYNTAX score $(\mathrm{r}=0.124, \mathrm{P}=0.108)$, hyperhomocysteinemia was $(\mathrm{r}=0.238, \mathrm{P}=0.002)$. The SYNTAX score was in turn negatively correlated with the adropin level ( $\mathrm{r}=-0.181, \mathrm{P}=0.018)$. There is thus evidence suggesting that serum adropin, while negatively associated with serum homocysteine, could predict coronary atherosclerosis quantified with SYNTAX score. Further pairwise correlation analyses revealed that SYNTAX score was significantly associated with age $(r=0.183, P=0.017)$ and serum creatinine $(r=0.364, P<0.001)$.

Generalized structural equation modeling for hyperhomocysteinemia and SYNTAX score. In view of the relatively large number of predictors considered in the confirmatory analysis with gSEM, a backward elimination routine (with $\mathrm{P} \geq 0.05$ as the criterion for variable elimination) was implemented to identify which demographic, clinical and angiographic variables were significantly associated with HyperHCY and SYNTAX, while considering the effect of serum adropin level. Extra consideration was taken when a large effect size was found, despite its statistical non-significance. The standard errors of the estimated effects were corrected with the robust statistical procedure. This ensured that the constructed gSEM was not sensitive to invalid modeling assumptions, particularly when there were potential outliers that could not be well accommodated with any parametric framework.

The final gSEM identified adropin and creatinine as the significant predictors of HyperHCY, and indicated that adropin 
Table II. Generalized structural equation modelling of SYNTAX score and hyperhomocysteinemia (homocysteine $>15 \mu$ mol/l).

A, SYNTAX score (SYNTAX sub-model)

\begin{tabular}{|c|c|c|c|}
\hline Variable & Coefficient & Robust $95 \%$ CI & P-value \\
\hline Adropin (pg/ml) & -0.04 & -0.06 to -0.01 & 0.019 \\
\hline \multicolumn{4}{|c|}{ Hyperhomocysteinemia } \\
\hline No & Reference & Reference & \\
\hline Yes & 4.71 & 1.39 to 8.03 & 0.005 \\
\hline Age (years) & 0.19 & 0.04 to 0.34 & 0.012 \\
\hline
\end{tabular}

B, Hyperhomocyteinemia (HyperHCY sub-model)

\begin{tabular}{lcc}
\hline Variable & AOR & Robust 95\% CI \\
\hline Adropin $(\mathrm{pg} / \mathrm{ml})$ & 0.95 & 0.93 to 0.98 \\
Creatinine $(\mu \mathrm{mol} / \mathrm{l})$ & 1.02 & 1.00 to 1.04 \\
Hypertension & & $\mathbf{0 . 0 0 2}$ \\
Without & Reference & $\mathbf{0 . 0 4 6}$ \\
With & 1.87 & 0.71 to 4.91 \\
\hline
\end{tabular}

AOR, adjusted odds ratio; CI, confidence interval. $\mathrm{P}$-values in bold are considered significant $(\mathrm{P}<0.05)$.

and HyperHCY affected the SYNTAX score (Table II). As revealed in the HyperHCY sub-model, adropin had a negative impact [adjusted odds ratio (AOR): 0.95, 95\% confidence interval $(\mathrm{CI}): 0.93$ to $0.98 ; \mathrm{P}=0.002)$ on HyperHCY and this conformed with the earlier pairwise correlation analysis. However, a higher level of creatinine (AOR: 1.02, 95\% CI: 1.00 to $1.04 ; \mathrm{P}=0.046$ ) was significantly associated with HyperHCY. It is worthy of note that hypertension was retained in the sub-model in view of its large effect size. When other variables were comparable, CAD patients suffering from hypertension were 1.87 -fold more likely to have hyperhomocysteinemia when compared with those without hypertension, although the effect was non-significant.

Adropin and HyperHCY were significantly associated with SYNTAX when adjusting for the effect of age, as revealed in the SYNTAX sub-model (Table II). All other variables being comparable, a unit increase in serum adropin level $(\mathrm{pg} / \mathrm{ml})$ would reduce the SYNTAX score by 0.04 units (95\% CI: -0.06 to $-0.01 ; \mathrm{P}=0.019)$. Patients with hyperhomocysteinemia had a significantly higher average SYNTAX score of 4.71 units $(95 \%$ CI: 1.39 to $8.03 ; \mathrm{P}=0.005)$, when compared with those not suffering from the condition. Finally, as the CAD patients aged their SYNTAX score was expected to increase (coefficient: $0.19,95 \%$ CI: 0.04 to $0.34 ; \mathrm{P}=0.012$ ). The analyses with gSEM confirmed that the study hypotheses were valid. While serum adropin was significantly associated with hyperhomocysteinemia, both low adropin level and hyperhomocysteinemia were significantly associated with coronary atherosclerosis.

\section{Discussion}

This study prospectively evaluated the association between serum homocysteine level and serum adropin level in patients with CAD, and identified the significant association of the two molecules with the severity of coronary atherosclerosis as represented by the SYNTAX score. The results showed that a low serum adropin level was significantly associated with hyperhomocysteinemia. Patients with hyperhomocysteinemia had a higher SYNTAX score, indicating more severe coronary atherosclerosis. By contrast, the serum adropin level was negatively correlated with SYNTAX score, suggesting a possible protective effect of serum adropin against coronary atherosclerosis.

Homocysteine is an intermediate formed during the metabolism of methionine, which is metabolized through vitamin-dependent pathways including remethylation (requiring folate and vitamin $\mathrm{B}_{12}$ ) back to methionine and trans-sulfuration (requiring vitamin $\mathrm{B}_{12}$ ) to cysteine and taurine (14). Studies have reported that the insufficiency of such vitamins in the body correlates with hyperhomocysteinemia, which could induce atherosclerosis and cardiovascular diseases (23-25). Homocysteinemia increases the vascular oxidative burden (26), activates inflammatory mediator of nuclear factor- $\kappa \mathrm{B}(\mathrm{NF}-\kappa \mathrm{B})(27)$, raises the endothelin-1 (ET-1) level, reduces coupled eNOS but increases the uncoupled form of eNOS, resulting in the excessive production of superoxide radicals instead of nitric oxide (28). These actions support the 'atherosclerosis hypothesis' of homocysteine from the intrinsic pathological mechanisms.

However, some clinical trials failed to demonstrate that homocysteine-lowering treatment with folic acid and vitamin $B_{12}$, and vitamin $B_{6}$ could reduce early atherosclerosis and the risk of recurrent cardiovascular events in patients with cardiovascular diseases (29-31). One possible explanation for the negative results could be that the study populations had a sufficiently folate-rich diet. Thus, any further treatment 
with such vitamins would provide no additional benefit. In addition, high-dose folic acid increases circulating but not intracellular 5-methyltetrahydrofolate, which plays a crucial role in homocysteine metabolism. Therefore, in spite of the uncertainty of the effectiveness of pharmacological treatment targeting the homocysteine level in patients with cardiovascular diseases, hyperhomocysteinemia was hypothesized to be associated with increased atherosclerotic and cardiovascular risk. As shown in the above confirmatory analysis, patients with hyperhomocysteinemia indeed had more severe coronary atherosclerosis, presenting with a higher SYNTAX score.

As a newly identified secreted protein with an impact on energy homeostasis, the primary effects of adropin are associated with glucose consumption, lipid metabolism and insulin sensitivity. A previous study demonstrated the beneficial effects of adropin on the regulation of eNOS bioactivity and endothelial function (16). Adropin is able to stimulate Akt Ser473 and endothelial nitric oxide synthase Ser1177 phosphorylation, thus resulting in the upregulation of nitric oxide formation and bioavailability (16). An epidemiological study reported that patients with cardiac syndrome $\mathrm{X}$ had a lower serum adropin level and plasma nitrite/nitrate level than healthy subjects, and the adropin level was positively correlated with nitrite/nitrate level (32). In a study of patients with type 2 diabetes, individuals with endothelial dysfunction had a lower plasma adropin level than individuals without endothelial dysfunction, thus suggesting the protective role of adropin in endothelial function (33). As such, given the adverse effects of homocysteine on endothelial function, adropin may antagonize homocysteine to protect endothelial function.

Insulin resistance may be a possible link between homocysteine and adropin. The association of hyperhomocysteinemia with insulin resistance has been demonstrated by several epidemiological and animal studies (34-36). Oxidative stress caused by hyperhomocysteinemia could interrupt insulin signaling and impair insulin action (37). A recent study demonstrated that hyperhomocysteinemia promoted insulin resistance by inducing endoplasmic reticulum stress, activating c-Jun $\mathrm{N}$-terminal kinase to stimulate proinflammatory cytokine production and facilitating macrophage infiltration (38). Due to its attenuating effect on insulin resistance, adropin may be a useful antagonist of homocysteine, playing a role in the prevention of oxidative stress and the further progression of atherogenesis.

In addition, the present study showed that creatinine was significantly associated with hyperhomocysteinemia, which in turn affected coronary atherosclerosis as represented by the SYNTAX score. The metabolism and clearance of homocysteine is mostly completed in the kidney, suggesting a potentially close association between homocysteine and renal function $(3,39)$. Renal function as evaluated using creatinine levels and the glomerular filtration rate has already been recognized a risk factor for the severity of CAD in a previous study (40).

The findings of the present study must be interpreted in light of the study's limitations. The reported results were based on a relatively small patient cohort. In addition, the evaluation of the association between homocysteine and adropin was conducted in patients with CAD. As such, the results might not be applicable to healthy subjects or the general population at large. Further confirmatory studies must be conducted to determine if the significant correlation between homocysteine and adropin could indeed be causal in nature.

In conclusion, a low serum adropin level was significantly associated with hyperhomocysteinemia in patients with CAD. In addition, patients with hyperhomocysteinemia had more severe coronary atherosclerosis presenting with a higher SYNTAX score. Also, the negative correlation between serum adropin level and SYNTAX score suggests that adropin potentially provides a protective effect against coronary atherosclerosis.

\section{Acknowledgements}

This study was supported by the Youth Natural Science Fund of Soochow University (grant number SDY2013A32) and Research Fund of the Second Affiliated Hospital of Soochow University (grant number SDFEYGJ1405). The authors gratefully acknowledge the assistance of Mr Hui Li, Mr Chun-Lai Shao, Mr Jian-Liang Jiang and Miss Jian-Feng Li with patient recruitment.

\section{References}

1. Veeranna V, Zalawadiya SK, Nirai A, Pradhan J, Ference B, Burack RC, Jacob S and Afonso L: Homocysteine and reclassification of cardiovascular disease risk. J Am Coll Cardiol 58: 1025-1033, 2011.

2. Mayer EL, Jacobsen DW and Robinson K: Homocysteine and coronary atherosclerosis. J Am Coll Cardiol 27: 517-527, 1996.

3. Schaffer A, Verdoia M, Cassetti E, Marino P, Suryapranata H and De Luca G; Novara Atherosclerosis Study Group (NAS): Relationship between homocysteine and coronary artery disease. Results from a large prospective cohort study. Thromb Res 134: 288-293, 2014.

4. Anderson JL, Muhlestein JB, Horne BD, Carlquist JF, Bair TL, Madsen TE and Pearson RR: Plasma homocysteine predicts mortality independently of traditional risk factors and C-reactive protein in patients with angiographically defined coronary artery disease. Circulation 102: 1227-1232, 2000.

5. Sun Q, Jia X, Gao J, Mou W, Tong H, Wen X and Tian Y: Association of serum homocysteine levels with the severity and calcification of coronary atherosclerotic plaques detected by coronary CT angiography. Int Angiol 33: 316-323, 2014.

6. Shenoy V, Mehendale V, Prabhu K, Shetty R and Rao P: Correlation of serum homocysteine levels with the severity of coronary artery disease. Indian J Clin Biochem 29: 339-344, 2014.

7. Mirhosseini SJ, Forouzannia SK, Mirhosseini SA, Ali-Hassan-Sayegh S and Mozayan MR: Intra-operative grading of coronary artery atherosclerosis associated with homocysteine levels in postmenopausal women undergoing elective off-pump CABG surgery. Niger Med J 53: 192-195, 2012.

8. Parthasarathy S. Oxidation of low-density lipoprotein by thiol compounds leads to its recognition by the acetyl LDL receptor. Biochim Biophys Acta 917: 337-340, 1987.

9. Zou T, Yang W, Hou Z and Yang J: Homocysteine enhances cell proliferation in vascular smooth muscle cells: Role of p38 MAPK and p47phox. Acta Biochim Biophys Sin (Shanghai) 42: 908-915, 2010.

10. Shirpoor A, Salami S, Khadem Ansari MH, Ilkhanizadeh B and Abdollahzadeh N: Ethanol promotes rat aortic vascular smooth muscle cell proliferation via increase of homocysteine and oxidized low-density lipoprotein. J Cardiol 62: 374-378, 2013.

11. Woo KS, Chook P, Lolin YI, Cheung AS, Chan LT, Sun YY, Sanderson JE, Metreweli C and Celermajer DS: Hyperhomocyst(e) inemia is a risk factor for arterial endothelial dysfunction in humans. Circulation 96: 2542-2544, 1997.

12. Snehalatha C, Ramachandran A, Satyavani K, Sivasankari S, Sathyamurthy I and Viswanathan V: Plasma homocysteine concentration and coronary artery disease in Asian Indians. J Assoc Physicians India 50: 1229-1231, 2002. 
13. Boufidou AI, Makedou AD, Adamidis DN, Karvounis HI, Gourassas JT, Kesidis HT, Makedou KG, Papadopoulos CE, Parharidis GE and Louridas GE: Association between plasma homocysteine levels and coronary artery disease: A population-based study in northern Greece. Curr Med Res Opin 20: $175-180,2004$

14. Kaul S, Zadeh AA and Shah PK. Homocysteine hypothesis for atherothrombotic cardiovascular disease: Not validated. J Am Coll Cardiol 48: 914-923, 2006.

15. Kumar KG, Trevaskis JL, Lam DD, Sutton GM, Koza RA, Chouljenko VN, Kousoulas KG, Rogers PM, Kesterson RA, Thearle M, et al: Identification of adropin as a secreted factor linking dietary macronutrient intake with energy homeostasis and lipid metabolism. Cell Metab 8: 168-481, 2008.

16. Lovren F, Pan Y, Quan A, Singh KK, Shukla PC, Gupta M, Al-Omran $\mathrm{M}$, Teoh $\mathrm{H}$ and Verma S: Adropin is a novel regulator of endothelial function. Circulation 122 (Suppl 1): S185-S192, 2010.

17. Ganesh Kumar K, Zhang J, Gao S, Rossi J, McGuinness OP, Halem HH, Culler MD, Mynatt RL and Butler AA: Adropin deficiency is associated with increased adiposity and insulin resistance. Obesity (Silver Spring) 20: 1394-1402, 2012.

18. Yu HY, Zhao P, Wu MC, Liu J and Yin W: Serum adropin levels are decreased in patients with acute myocardial infarction. Regul Pept 190-191: 46-49, 2014.

19. Wu L, Fang J, Chen L, Zhao Z, Luo Y, Lin C and Fan L: Low serum adropin is associated with coronary atherosclerosis in type 2 diabetic and non-diabetic patients. Clin Chem Lab Med 52: 751-758, 2014

20. Rabe-Hesketh S, Skorndal A and Pickles A. Generalized multilevel structural equation modeling. Psychometrika 69: 167-190, 2004

21. El Oudi M, Bouguerra C, Aouni Z, Mazigh C, Bellaaj R and Machghoul S: Homocysteine and inflammatory biomarkers plasma levels and severity of acute coronary syndrome. Ann Biol Clin (Paris) 69: 175-180, 2011

22. Wu Y, Yang L and Zhong L. Decreased serum levels of thioredoxin in patients with coronary artery disease plus hyperhomocysteinemia is strongly associated with the disease severity. Atherosclerosis 212: 351-355, 2010.

23. Bertoia ML, Pai JK, Cooke JP, Joosten MM, Mittleman MA, Rimm EB and Mukamal KJ: Plasma homocysteine, dietary $B$ vitamins, betaine and choline and risk of peripheral artery disease. Atherosclerosis 235: 94-101, 2014.

24. Debreceni B and Debreceni L. The role of homocysteine-lowering $\mathrm{B}$-vitamins in the primary prevention of cardiovascular disease. Cardiovasc Ther 32: 130-138, 2014.

25. Boushey CJ, Beresford SA, Omenn GS and Motulsky AG: A quantitative assessment of plasma homocysteine as a risk factor for vascular disease. Probable benefits of increasing folic acid intakes. JAMA 274: 1049-1057, 1995

26. Ungvari Z, Csiszar A, Edwards JG, Kaminski PM, Wolin MS, Kaley $\mathrm{G}$ and Koller A: Increased superoxide production in coronary arteries in hyperhomocysteinemia: Role of tumor necrosis factor-alpha, NAD(P)H oxidase and inducible nitric oxide synthase. Arterioscler Thromb Vasc Biol 23: 418-424, 2003 .
27. Au-Yeung KK, Woo CW, Sung FL, Yip JC, Siow YL and OK: Hyperhomocysteinemia activates nuclear factor-kappaB in endothelial cells via oxidative stress. Circ Res 94: 28-36, 2004.

28. Shirodaria C, Antoniades C, Lee J, Jackson CE, Robson MD, Francis JM, Moat SJ, Ratnatunga C, Pillai R, Refsum H, et al: Global improvement of vascular function and redox state with low-dose folic acid: Implications for folate therapy in patients with coronary artery disease. Circulation 115: 2262-2270, 2007.

29. Cacciapuoti F: Lowering homocysteine levels with folic acid and B-vitamins do not reduce early atherosclerosis, but could interfere with cognitive decline and Alzheimer's disease. J Thromb Thrombolysis 36: 258-262, 2013.

30. Bønaa KH, Njølstad I, Ueland PM, Schirmer H, Tverdal A Steigen T, Wang H, Nordrehaug JE, Arnesen E and Rasmussen K; NORVIT Trial Investigators: Homocysteine lowering and cardiovascular events after acute myocardial infarction. N Engl J Med 354: 1578-1588, 2006.

31. Lonn E, Yusuf S, Arnold MJ, Sheridan P, Pogue J, Micks M, McQueen MJ, Probstfield J, Fodor G, Held C, et al: Homocysteine lowering with folic acid and B vitamins in vascular disease. N Engl J Med 354: 1567-1577, 2006.

32. Celik A, Balin M, Kobat MA, Erdem K, Baydas A, Bulut M, Altas Y, Aydin S and Aydin S: Deficiency of a new protein associated with cardiac syndrome $\mathrm{X}$; called adropin. Cardiovasc Ther 31: 174-178, 2013.

33. Topuz M, Celik A, Aslantas T, Demir AK, Aydin S and Aydin S: Plasma adropin levels predict endothelial dysfunction like flow-mediated dilatation in patients with type 2 diabetes mellitus. J Investig Med 61: 1161-1164, 2013.

34. Sanchez-Margalet V, Valle M, Ruz FJ, Gascon F, Mateo J and Goberna R: Elevated plasma total homocysteine levels in hyperinsulinemic obese subjects. J Nutr Biochem 13: 75-79, 2002.

35. Martos R, Valle M, Morales R, Cañete R, Gavilan MI and Sánchez-Margalet V: Hyperhomocysteinemia correlates with insulin resistance and low-grade systemic inflammation in obese prepubertal children. Metabolism 55: 72-77, 2006.

36. Pravenec M, Kozich V, Krijt J, Sokolová J, Zídek V, Landa V, Simáková M, Mlejnek P, Silhavy J, Oliyarnyk O, et al: Folate deficiency is associated with oxidative stress, increased blood pressure and insulin resistance in spontaneously hypertensive rats. Am J Hypertens 26: 135-140, 2013.

37. Najib S and Sánchez-Margalet V. Homocysteine thiolactone inhibits insulin signaling and glutathione has a protective effect. J Mol Endocrinol 27: 85-91, 2001.

38. Li Y, Zhang H, Jiang C, Xu M, Pang Y, Feng J, Xiang X, Kong W, $\mathrm{Xu}$ G, Li Y and Wang X: Hyperhomocysteinemia promotes insulin resistance by inducing endoplasmic reticulum stress in adipose tissue. J Biol Chem 288: 9583-9592, 2013.

39. Chua S, Wu CJ, Chang HW, Hang CL, Chen CJ, Yang CH and Yip HK: Impact of elevated plasma total homocysteine concentration on coronary atherosclerosis in Chinese patients with acute myocardial infarction undergoing primary coronary intervention. Int Heart J 46: 181-193, 2005.

40. Wang J, Sim AS, Wang XL, Salonikas C, Moriatis M, Naidoo D and Wilcken DE: Relations between markers of renal function, coronary risk factors and the occurrence and severity of coronary artery disease. Atherosclerosis 197: 853-859, 2008. 